\title{
Um mapeamento de pesquisas sobre atividades de Modelagem Matemática desenvolvidas de forma online
}

\author{
Rhômulo Oliveira Menezes \\ Marcelo Almeida Bairral
}

\begin{abstract}
Resumo: Neste artigo apresenta-se um mapeamento de pesquisas que pautaram o desenvolvimento de atividades de Modelagem Matemática de forma online. Os aspectos metodológicos de busca, seleção e análise dos trabalhos seguiram as orientações de Randolph (2009). Inicialmente optamos por um mapeamento em dissertações e teses compreendidas no recorte temporal de 2001 a 2018, que foram analisadas por Menezes (2019) e publicado nos Anais da $39^{a}$ Reunião Nacional da Associação Nacional de Pós-Graduação e Pesquisa em Educação. Para este texto, ampliamos os tipos de trabalhos considerados selecionando, também, artigos de periódicos e de anais de eventos, nacionais e internacionais da área de Educação Matemática. Esse mapeamento aponta que existem poucos trabalhos sobre atividades de Modelagem Matemática desenvolvidas de forma online, e desses poucos trabalhos, menor ainda é o número daqueles em que as pesquisas investigaram atividades de Modelagem Matemática desenvolvidas de forma online e totalmente síncrona, revelando esse contexto como promissor para o desenvolvimento de novas pesquisas.
\end{abstract}

Palavras-chave: Mapeamento. Produção Científica. Modelagem Matemática. Atividade online.

\section{A mapping of research on Mathematical Modeling activities}

Rhômulo Oliveira Menezes Doutor em Educação em Ciências e Matemáticas pela Universidade Federal do Pará (UFPA). Professor da Secretaria de Educação do Estado do Pará (Seduc-

PA), Capanema, Pará, Brasil.

Dhttp://orcid.org/0000-0001-9042-8323 $\triangle$ rrhomulo.menezes4542@escola.seduc .pa.gov.br

Marcelo Almeida Bairral Doutor em Educação Matemática pela Universidade de Barcelona (UB) Professor Titular da Universidade Federal Rural do Rio de Janeiro (UFRRJ), Seropédica, RJ, Brasil.

(D) http://orcid.org/0000-0002-5432-9261 $\triangle$ mbairral@ufrri.b

Recebido em 11/04/2021 Aceito em 13/05/2021 Publicado em 26/07/2021 \section{developed online}

Abstract: This article presents a mapping of research that guided the development of Mathematical Modeling activities online. The methodological aspects of search selection and analysis of the works followed the guidelines of Randolph (2009). Initially we opted for a mapping in dissertations and theses included in the time frame from 2001 to 2018, which were analyzed by Menezes (2019) and published in the Proceedings of the 39th National Meeting of the National Association of Graduate Studies and Research in Education. For this text we expanded the types of work considered by also selecting articles from national and international journals and event proceedings in the field of Mathematics Education. This mapping shows that there are few works on Mathematical Modeling activities developed online and of these few works even smaller is the number of those in which research investigated Mathematical Modeling activities developed online and fully synchronous revealing this context as promising for the development of new research.

Keywords: Mapping. Scientific Production. Mathematical Modeling. Online Activity.

\section{Una cartografía de investigaciones sobre actividades de modelización matemática desarrollado en línea}

\begin{abstract}
Resumen: Este artículo presenta una cartografía de investigaciones con énfasis en el desarrollo de actividades de Modelado Matemático en línea. Los aspectos metodológicos de búsqueda, selección y análisis de los trabajos siguieron las pautas de Randolph (2009). Inicialmente, optamos por una cartografía en disertaciones y tesis incluidas en el marco temporal de 2001 a 2018, las cuales fueron analizadas por Menezes (2019) y publicadas en las Actas del $39^{\circ}$ Encuentro Nacional de la Asociación Nacional de Postgrados e Investigaciones en Educación. Para este artículo, ampliamos los tipos de trabajo
\end{abstract}


considerados, seleccionando también artículos de revistas nacionales e internacionales, y actas de eventos en el campo de la Educación Matemática. Esta cartografía muestra que hay pocos trabajos sobre actividades de Modelación Matemática desarrollados en línea, y de estos pocos estudios, aún menor es el número de aquellos en los que los investigadores investigaron actividades de Modelización Matemática desarrolladas en línea y totalmente sincrónicas, revelando este contexto como prometedor para el desarrollo de nuevas investigaciones.

Palabras clave: Cartografía. Producción Científica. Modelización matemática. Actividad en línea.

\title{
1 Introdução
}

Este artigo remete ao início de uma pesquisa de doutorado em Educação em Ciências e Matemática, que tinha como tema de investigação a Modelagem Matemática desenvolvida de forma online. Dessa forma para delinear o objeto de pesquisa fizemos um mapeamento para identificar e reconhecer o campo em que o objeto estava inserido. Sobre essa fase inicial de pesquisa Biembengut (2008) considera que

\begin{abstract}
trata-se de um esforço inicial não apenas para evitarmos o levantamento de dados desnecessários, mas principalmente, para vermos emergir um primeiro mapa, uma estrutura não linear que indique relações, hierarquias, proporções entre múltiplos elementos: teorias, pessoas direta e indiretamente envolvidas, dados empíricos, dentre outros. (BIEMBENGUT, 2008, p. 79).
\end{abstract}

Com a criação do catálogo de teses e dissertações da Coordenação de Aperfeiçoamento de Pessoal de Nível Superior (Capes, MEC, Brasil) e o incremento de periódicos online e de publicações de Anais de congressos, dentre outros, o uso de mapeamento tem sido um momento importante na construção da revisão de literatura inicial de uma investigação que permite ao pesquisador reconhecer seu objeto e formular uma questão de investigação.

Dessa forma, o objetivo do artigo é descrever um mapeamento de pesquisas que abordaram o desenvolvimento de atividades de Modelagem Matemática de forma online. Foram selecionados trabalhos que apresentavam o desenvolvimento de cursos ou de atividades de Modelagem Matemática mediadas de forma online. Nesses trabalhos focamos na identificação da questão de investigação, da perspectiva de Modelagem Matemática adotada, do tipo de ambiente virtual de aprendizagem utilizado, do público-alvo, e do tipo de encontro (síncrono, assíncrono ou híbrido ${ }^{1}$ ).

\footnotetext{
${ }^{1}$ Mercado (2009) destaca três tipos de comunicação na educação online: 1) síncrona, as interações são em tempo real; 2) assíncrona, as interações são em tempo diferido (não real); e 3) híbrida, utiliza a comunicação síncrona e assíncrona.
} 
Sobre atividades de Modelagem Matemática desenvolvidas de forma online, Menezes $(2019)^{2}$ fez um mapeamento que compreendeu dissertações e teses de 2001 a 2018. Desse período foram selecionados seis trabalhos: Malheiros (2008a), Santana (2010), Costa (2010), Ferreira (2010), Pereira (2015) e Bustamante (2016).

Os resultados desse mapeamento mostraram que as atividades de Modelagem Matemática em três trabalhos - Costa (2010), Ferreira (2010) e Pereira (2015) - foram desenvolvidas na Plataforma Moodle. Saindo desse escopo tem os trabalhos de Malheiros (2008a) que usou o AVA Tecnologia da Informação no Desenvolvimento da Internet Avançada Aprendizado Eletrônica (TIDIA-Ae ${ }^{3}$ ), de Santana (2010) que usou um AVA de um curso de Matemática à distância, e de Bustamante (2016) que usou o Facebook como AVA.

Já em relação ao público-alvo dessas atividades, foram em sua maioria professores que ensinam matemática em formação continuada com encontros do tipo híbrido, síncronos e assíncronos, como informado em Malheiros (2008a), Ferreira (2010), Pereira (2015) e Bustamante (2016). Diferentemente dos trabalhos de Santana (2010) e Costa (2010), nos quais o público-alvo foram professores que ensinam matemática em formação inicial e a comunicação estabelecida foi por meio de encontros assíncronos. Dos trabalhos mapeados por Menezes (2019) foi possível destacar características sobre:

- as perspectivas de Modelagem Matemática, que no contexto online variaram de trabalho para trabalho. Em Malheiros (2008a) as atividades foram desenvolvidas como Projetos de Modelagem. Em Santana (2010) e Pereira (2015) a Modelagem Matemática foi entendida, respectivamente, como um ambiente (BARBOSA, 2003, 2006) e alternativa pedagógica (BARBOSA, 2004). Com foco na aliança entre teoria e prática, o trabalho de Costa (2010) norteou a pesquisa a partir de Bassanezi (2002). Ferreira (2010) desenvolveu suas atividades segundo um conjunto de procedimentos (BURAK, 1987, 2004), e Bustamante (2016), de acordo com a concepção de Borba e Villarreal (2005) de gerar ou propor problemas matemáticos;

- o público-alvo das atividades de Modelagem Matemática, a maioria era de professores em formação continuada, somente Santana (2010) tinha como público graduandos em

\footnotetext{
2 Primeiro autor deste artigo

3 Projeto financiado pela Fundação de Amparo à Pesquisa do Estado de São Paulo (FAPESP). Programa de Pesquisa e Desenvolvimento nas diversas facetas da tecnologia de informação, telecomunicações e redes de computadores associadas com a Internet Avançada, sendo subdividido nos projetos Kyatera, Incubadora Virtual e Aprendizado eletrônico (Ae) (MALHEIROS, 2008a, p. 17).
} 
formação inicial, e no trabalho de Costa (2010) no qual graduandos participavam em conjunto com outros pesquisadores e professores;

- o AVA, a maioria das atividades foram desenvolvidas em ambientes voltados para o ensino e aprendizagem, sendo diferente em Bustamante (2016), que optou por fazer uso de grupos fechados no Facebook;

- os tipos de encontros, a maioria dos trabalhos relata encontros híbridos, síncronos e assíncronos, destacando-se Santana (2010) e Costa (2010) que realizaram atividades apenas por encontros assíncronos.

Como em Menezes (2019) o mapeamento foi realizado em dissertações e teses, e poucos trabalhos foram encontradas de 2001 a 2018, ampliamos as buscas incluindo trabalhos oriundos de periódicos e de anais de eventos da área de Educação Matemática. A seguir apresentamos como se deu a coleta, organização e as análises desses trabalhos.

\section{Coleta, organização e análise de artigos de periódicos e de anais de eventos}

De acordo com Biembengut (2008) para uma primeira identificação das produções existentes, estabelecemos as palavras-chaves ou tema central do objeto da pesquisa e a seguir, identificamos possíveis fontes. Como fontes a autora aponta a internet, entendendo essa rede internacional de computadores como um meio especial no qual existe uma variedade de sítios eletrônicos que podem servir como primeiro caminho.

Escolhemos como primeiro caminho o site da Sociedade Brasileira de Educação Matemática (SBEM)4, que dispunha de uma lista de 17 (dezessete) periódicos nacionais e 17 (dezessete) internacionais ${ }^{5}$. Existem mais periódicos na área de Educação Matemática, que não estão citados no site da SBEM, no entanto, consideramos o recorte feito pela maior organização da área no Brasil como suficiente para dar início a esta revisão de literatura. Essa escolha além de incentivar o uso desse site como consulta também reitera a excelência dos periódicos listados, sendo bem avaliados com relação Qualis/Capes que podem ser consultados no site da Plataforma Sucupira, por exemplo, o periódico Boletim de Educação Matemática (online) possui estrato A1, o periódico Educação Matemática Pesquisa (online) possui estrato A2, o periódico ZDM Mathematics Education possui estrato A1; todas essas avaliações na área de Ensino.

\footnotetext{
${ }^{4}$ http://www.sbembrasil.org.br/sbembrasil/index.php/materiais/periodicos

${ }^{5} \mathrm{~A}$ busca por periódicos foi iniciada às duas horas e cinquenta e cinco minutos do dia 26/01/2019 e finalizada às vinte e três horas e vinte sete minutos do dia 03/03/2019.
} 
Os sites dos trinta e quatro periódicos foram acessados observando nos sumários das edições, títulos de artigos em periódicos nacionais que continham a palavra "modelagem", "modelação" ou "modelo" e em periódicos internacionais que continham o prefixo "mod" por englobar referências à palavra "modelagem", "modelação" ou "modelo" escritas em outros idiomas, como: "modeling", "modelación", "modelaje", "modelización" ou "model”. Nos periódicos nacionais foram encontrados 187 (cento e oitenta e sete) artigos em 14 (quatorze) periódicos, e nos internacionais foram encontrados 223 (duzentos e vinte e três) artigos em 13 (treze) periódicos. Considerando as indicações de Randolph (2009)6 apontadas no mapeamento de Menezes (2019), analisamos o título, resumo e palavras-chave dos 410 (quatrocentos e dez artigos) ${ }^{7}$, selecionando um total de 06 (seis) artigos, como destacado no Quadro 1.

Quadro 1: Artigos de periódicos

\begin{tabular}{|c|c|c|c|}
\hline TIPO & TÍTULO DO ARTIGO & $\begin{array}{l}\text { AUTORES } \\
\text { (ANO) }\end{array}$ & $\begin{array}{c}\text { PERIÓDICOS } \\
\text { (VOLUME, NÚMERO) }\end{array}$ \\
\hline \multirow{3}{*}{ Nacional } & $\begin{array}{c}\text { Educação Matemática online: a elaboração de } \\
\text { projetos de Modelagem }\end{array}$ & $\begin{array}{l}\text { Malheiros } \\
\text { (2008b) }\end{array}$ & $\begin{array}{l}\text { Bolema - Revista } \\
\text { Boletim de Educação } \\
\text { Matemática (v. 21, n. } \\
\text { 30) }\end{array}$ \\
\hline & $\begin{array}{l}\text { Educação Matemática online: a elaboração de } \\
\text { projetos de modelagem }\end{array}$ & $\begin{array}{l}\text { Matucheski } \\
\quad(2012)\end{array}$ & $\begin{array}{c}\text { Bolema - Revista } \\
\text { Boletim de Educação } \\
\text { Matemática (v. 26, n. } \\
\text { 42B) }\end{array}$ \\
\hline & $\begin{array}{l}\text { A escrita e o pensamento matemático no } \\
\text { ambiente virtual utilizando a modelagem } \\
\text { matemática: experiência de uma turma de } 9^{\circ} \text { ano }\end{array}$ & $\begin{array}{l}\text { Schroetter et } \\
\text { al. (2016) }\end{array}$ & $\begin{array}{l}\text { Educação Matemática } \\
\text { Pesquisa (V. 18, n. 1) }\end{array}$ \\
\hline \multirow{3}{*}{ Internacional } & $\begin{array}{c}\text { Developing a mathematical modeling course in a } \\
\text { virtual learning environment }\end{array}$ & $\begin{array}{l}\text { Orey; Rosa } \\
\quad(2018)\end{array}$ & \multirow{2}{*}{$\begin{array}{c}\text { ZDM - Mathematics } \\
\text { Education (V. } 50, \text { Issue } \\
1-2)\end{array}$} \\
\hline & $\begin{array}{l}\text { Expansive movements in the development of } \\
\text { mathematical modeling: analysis from an Activity } \\
\text { Theory perspective }\end{array}$ & $\begin{array}{l}\text { Galleguillos; } \\
\text { Borba (2018) }\end{array}$ & \\
\hline & $\begin{array}{l}\text { Participación de profesores en un ambiente } \\
\text { de formación online. }\end{array}$ & $\begin{array}{l}\text { Parra- } \\
\text { Zapata et al. } \\
\quad(2018)\end{array}$ & $\begin{array}{l}\text { Educación Matemática } \\
\quad(\text { v. } 30, \text { n. 1) }\end{array}$ \\
\hline
\end{tabular}

Fonte: Elaboração própria

Algumas semelhanças entre os trabalhos já discutidos em Menezes (2019) e os periódicos incluídos na revisão de literatura foram observadas. Por exemplo, os artigos de Malheiros (2008b) e Matucheski (2012) publicados respectivamente nos volumes 21 e 26 da Bolema são resumos da tese de Malheiros (2008a). $O$ artigo de Galleguillos e Borba (2018), publicado no volume 50 do ZDM - Mathematics Education é um recorte da tese de Bustamante (2016). Considerando que as

\footnotetext{
${ }^{6}$ Randolph (2009) sugere que partindo de uma análise no título, resumo e palavras-chave pode-se incluir ou excluir trabalhos.

${ }^{7}$ Artigos datados no período de 1980-2018.
} 
atividades de Modelagem Matemática relatadas e analisadas nesses periódicos são as mesmas, mesmo que com focos de investigação distintos, não voltei a discuti-las nesta seção.

Assim, optamos pelos trabalhos de Schroetter et al. (2016), Orey e Rosa (2018), e ParraZapata et al. (2018), e conforme feito em Menezes (2019) para análise dos artigos de periódicos selecionados focamos na identificação da questão de investigação, da perspectiva de Modelagem Matemática adotada, do tipo de ambiente virtual de aprendizagem utilizado, do público-alvo, e do tipo de encontro, como delimitado no Quadro 2.

Quadro 2: Tópicos de análise dos artigos de periódicos

\begin{tabular}{|c|c|c|c|c|c|}
\hline $\begin{array}{l}\text { AUTOR } \\
\text { (ANO) }\end{array}$ & PROBLEMA OU OBJETIVO & $\begin{array}{c}\text { PERSPECTIVA DE } \\
\text { MM }^{8}\end{array}$ & AVA & PÚBLICO & $\begin{array}{c}\text { TIPO DE } \\
\text { ENCONTRO }\end{array}$ \\
\hline 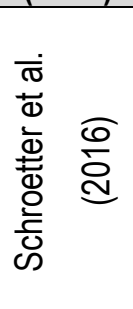 & $\begin{array}{l}\text { O presente artigo apresenta } \\
\text { o relato de um trabalho de } \\
\text { pesquisa utilizando a escrita } \\
\text { como ferramenta para o } \\
\text { desenvolvimento do processo } \\
\text { cognitivo da aprendizagem } \\
\text { em matemática. }\end{array}$ & $\begin{array}{c}\text { Metodologia de } \\
\text { ensino/aprendizagem } \\
\text {, Biembengut e Hein } \\
\text { (2000), } \\
\text { Stahl (2003), Meyer, } \\
\text { Caldeira e Malheiros } \\
\text { (2011) }\end{array}$ & e-mail9 & $\begin{array}{l}\text { Alunos do } 9^{\circ} \\
\text { ano do } \\
\text { Ensino } \\
\text { Fundamenta } \\
\quad \text { I }\end{array}$ & Assíncronos \\
\hline 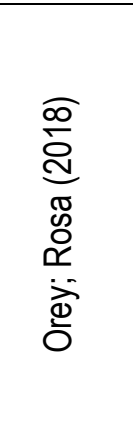 & $\begin{array}{c}\text { Como os recursos } \\
\text { tecnológicos disponíveis em } \\
\text { um Ambiente Virtual de } \\
\text { Aprendizagem (AVA) ajudam } \\
\text { os alunos a interagir e } \\
\text { desenvolver projetos } \\
\text { colaborativos de modelagem } \\
\text { matemática que os auxiliem } \\
\text { na solução de problemas que } \\
\text { enfrentam no dia a dia? }\end{array}$ & $\begin{array}{c}\text { Projetos de } \\
\text { Modelagem. Rosa } \\
(2000)\end{array}$ & Moodle & Graduandos & \multirow[t]{2}{*}{$\begin{array}{l}\text { Síncronos e } \\
\text { Assíncronos }\end{array}$} \\
\hline 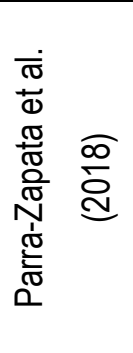 & $\begin{array}{c}\text { Este artigo analisa a } \\
\text { participação de um grupo de } \\
\text { professores em uma sessão } \\
\text { de modelagem matemática } \\
\text { em um espaço de } \\
\text { treinamento online. }\end{array}$ & $\begin{array}{l}\text { Alternativa para } \\
\text { promover, formas } \\
\text { diferentes de } \\
\text { participação; } \\
\text { desenvolver atitudes } \\
\text { criativa e crítica. } \\
\text { Parra-Zapata et al. } \\
\text { (2017) }\end{array}$ & $\begin{array}{l}\text { Wiz/Q } \\
e \\
\text { Moodle }\end{array}$ & Professores & \\
\hline
\end{tabular}

Fonte: Elaboração própria

Essa quantidade reduzida de artigos de periódicos de um universo de 410 (quatrocentos e dez) artigos confirmou as afirmativas feitas em Menezes (2019), de que quando focado no

\footnotetext{
8 Modelagem Matemática

9 Não é um AVA, mas na atividade relatada funcionou como um, no qual se estabeleceu entre professora e alunos uma comunicação assíncrona.

10 É uma plataforma de ensino e aprendizagem que permite conversar em tempo real com quem quiser e partilhar um quadro branco, onde todos podem escrever e desenhar. Também é possível fazer o upload de diversos tipos de documentos (.ppt, .doc, .pdf) e mostrá-los aos participantes da sessão. Todos podem colocar questões, escrever no quadro branco ou sobre os próprios documentos. A plataforma permite ainda que a sessões sejam gravadas.
} 
desenvolvimento de atividades de Modelagem Matemática no contexto online o número de trabalhos é reduzido. Assim reforçando a necessidade de mais pesquisas nesse viés temático.

Em relação aos anais de eventos nacionais e internacionais da área de Educação Matemática, a inclusão desses trabalhos justificou-se por entendermos os eventos como espaços em que há uma cobertura maior de participação de alunos de graduação, professores e pesquisadores. Resultando assim, em inúmeras comunicações e relatos de distintas linhas de investigação da área da Educação Matemática apresentados e publicados nos anais.

Dessa forma, optamos pelo maior evento de Modelagem Matemática no Brasil, a Conferência Nacional de Modelagem na Educação Matemática (CNMEM), e por eventos no âmbito nacional, o Encontro Nacional de Educação Matemática (ENEM), e internacional, Seminário Internacional de Pesquisa em Educação Matemática (SIPEM). Eventos nos quais a Modelagem Matemática possui um grupo de trabalho (GT10 - Modelagem Matemática), estabelecido pela SBEM desde 2001.

Os anais da CNMEM não se encontram disponíveis todos em um único lugar, por isso, 0 acesso a eles se deu no contato com integrantes do Grupo de Estudos e Pesquisas em Modelagem Matemática (GEMM), da Universidade Federal do Pará (UFPA). Foram consultados os anais das edições: I (1999), III (2003), IV (2005), V (2007), VI (2009), VII (2011), VIII (2013), IX (2015), X (2017). Não tivemos acesso aos anais da edição II (2001). Já os anais do ENEM e do SIPEM estão disponíveis no site da SBEM. Do ENEM foram considerados os anais das edições compreendidas entre I (1987) a XII (2016), e do SIPEM foram considerados os anais das edições compreendidas entre I (2000) a VI (2015).

Os anais da CNMEM até a edição X (2017) eram disponibilizados em CD-ROM, por isso cada trabalho que estava no formato PDF foi aberto e analisado título, resumo e palavras-chave. Nos anais do ENEM e do SIPEM encontramos nas primeiras edições arquivos únicos contendo todos os trabalhos dos respectivos eventos e nas edições mais recentes os anais são organizados por trabalho listados a partir do título do trabalho e dos autores nos sites dos eventos. No caso de arquivos únicos procuramos trabalhos usando a ferramenta busca e o prefixo 'mod' como feito para os periódicos, e no caso dos trabalhos organizados por sumários de títulos e autores usamos também a ferramenta busca do próprio navegador de internet e o prefixo 'mod'. Sobre esse último, quando encontrávamos alguma referência a Modelagem Matemática no título abríamos os arquivos e liamos o resumo e palavras-chave. Com essas buscas encontramos sete trabalhos sobre atividades de Modelagem Matemática desenvolvidas de forma online, conforme apresentado no Quadro 3. 
Quadro 3: Artigos de anais de eventos

\begin{tabular}{|c|c|c|c|}
\hline TIPO & TÍTULO DO ARTIGO & AUTORESI ANO & $\begin{array}{l}\text { EDIÇÃOl } \\
\text { EVENTO }\end{array}$ \\
\hline Internacional & Projetos de Modelagem em um curso de formação online & Malheiros (2009) & IV SIPEM \\
\hline \multirow{6}{*}{ Nacional } & $\begin{array}{c}\text { Considerações iniciais sobre um curso de } \\
\text { Modelagem Matemática em Ambientes Virtuais }\end{array}$ & $\begin{array}{l}\text { Ferreira; Burak } \\
(2009)\end{array}$ & VI CNMEM \\
\hline & $\begin{array}{l}\text { Modelagem Matemática - uma experiência com a } \\
\text { formação continuada de professores utilizando a } \\
\text { educação a distância online }\end{array}$ & $\begin{array}{l}\text { Ferreira; Burak } \\
\quad(2010)\end{array}$ & X ENEM \\
\hline & $\begin{array}{l}\text { Analisando aspectos da organização de um curso de } \\
\text { Modelagem à distância: uma contribuição para o debate } \\
\text { da pedagogia on-line na educação matemática }\end{array}$ & Santana (2013) & $\begin{array}{c}\text { VIII } \\
\text { CNMEM }\end{array}$ \\
\hline & $\begin{array}{c}\text { Proposta e Resolução de um problema de Modelagem } \\
\text { na } \\
\text { Educação Matemática online }\end{array}$ & $\begin{array}{l}\text { Galleguillos } \\
\text { (2016) }\end{array}$ & XII ENEM \\
\hline & $\begin{array}{c}\text { Propondo a Modelagem Matemática como um Ambiente } \\
\text { Virtual de Aprendizagem }\end{array}$ & $\begin{array}{l}\text { Freitas; Orey; } \\
\text { Rosa (2017) }\end{array}$ & \multirow{2}{*}{ X CNMEM } \\
\hline & $\begin{array}{l}\text { Modelagem Matemática no Ambiente Virtual de ensino e } \\
\text { aprendizagem: alguns encaminhamentos }\end{array}$ & $\begin{array}{l}\text { Borssoi; Silva; } \\
\text { Ferruzzi (2017) }\end{array}$ & \\
\hline
\end{tabular}

Fonte: Elaboração própria

Ao analisarmos os artigos de anais de eventos selecionados, encontramos interseções entre eles e outros trabalhos já discutidos em Menezes (2019) e nos artigos encontrados em periódicos. O artigo de Malheiros (2009) publicado nos anais do IV SIPEM é um recorte da tese de Malheiros (2008a), que também se relaciona com os artigos de Malheiros (2008b) e Matucheski (2012). Os artigos de Ferreira e Burak (2009) publicado na VI CNMEM e Ferreira e Burak (2010) publicado no X ENEM são recortes da dissertação de Ferreira (2010). O artigo de Santana (2013) publicado na VIII CNMEM é um recorte da dissertação de Santana (2010). $O$ artigo de Galleguillos (2016) é um recorte da dissertação de Bustamante (2016), que também se relaciona com o artigo de Galleguillos e Borba (2018). Assim, considerando que os artigos trazem novos olhares para as mesmas atividades de Modelagem Matemática desenvolvidas de forma online, que já foram discutidas anteriormente, resolvemos não os discutir de novo.

No artigo de Freitas, Orey e Rosa (2017) publicado na X CNMEM identificamos aproximações com o artigo de Orey e Rosa (2018), e acrescido a isso se trata de um artigo teórico, fugindo do escopo delimitado incialmente de discutir trabalhos que descrevessem atividades de Modelagem Matemática desenvolvidas de forma online. Assim, focamos na identificação da questão de investigação, da perspectiva de Modelagem Matemática adotada, do tipo de ambiente virtual de aprendizagem utilizado, do público-alvo, e do tipo de encontro no trabalho de Borssoi, Silva e Ferruzzi (2017) publicado na X CNMEM, conforme Quadro 4. 
Quadro 4: Tópicos de análise dos artigos de anais de eventos

\begin{tabular}{|c|c|c|c|c|c|}
\hline $\begin{array}{l}\text { AUTOR } \\
\text { ANO }\end{array}$ & PROBLEMA OU OBJETIVO & $\begin{array}{c}\text { PERSPECTIVA } \\
\text { DE MM }\end{array}$ & AVA & PÚBLICO & $\begin{array}{c}\text { TIPO DE } \\
\text { ENCONTRO }\end{array}$ \\
\hline 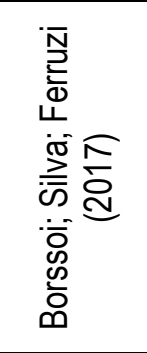 & $\begin{array}{l}\text { Discutir o desenvolvimento de } \\
\text { atividades de modelagem matemática } \\
\text { amparado pelo uso de recursos } \\
\text { tecnológicos, mais especificamente } \\
\text { sobre o uso de ambientes virtuais de } \\
\text { ensino e de aprendizagem, enquanto } \\
\text { aliadas para o ensino e aprendizagem } \\
\text { de conteúdos matemáticos. }\end{array}$ & $\begin{array}{c}\text { Fases da } \\
\text { Modelagem } \\
\text { Matemática, } \\
\text { Almeida, Silva e } \\
\text { Vertuan (2012) }\end{array}$ & Moodle & Graduandos & Assíncronos \\
\hline
\end{tabular}

Fonte: Elaboração própria

Ao considerar o exposto acerca da coleta e organização dos artigos de periódicos e dos artigos de anais de eventos, os trabalhos selecionados foram: Schroetter et al. (2016), Orey; Rosa (2018), Parra-Zapata et al. (2018) e Borssoi; Silva; Ferruzi (2017). Na seção seguinte apresentamos discussões desses trabalhos norteadas pelos itens destacados nos Quadros 2 e 4.

\section{Discussões dos artigos de periódicos e de anais de eventos segundo a perspectiva de MM, o tipo de AVA, o público-alvo e o tipo de encontro}

No mapeamento de teses e dissertações realizado em Menezes (2019) os resultados apontavam para pesquisas, que tinham como foco o trabalho com a formação inicial ou continuada de professores que ensinam matemática. Para o desenvolvimento das atividades realizava-se encontros híbridos ou assíncronos, em que o foco acabava sendo o planejamento e 0 desenvolvimento online de atividades para serem realizadas em aulas presenciais de Matemática.

Neste sentido, os trabalhos de Orey e Rosa (2018), Parra-Zapata et al. (2018) e Borssoi, Silva e Ferruzzi (2017) direcionaram suas atividades de Modelagem Matemática para professores em formação inicial ou continuada. Orey e Rosa (2018) e Borssoi, Silva e Ferruzzi (2017), com foco na formação inicial. Os autores da primeira obra descreveram o andamento de uma disciplina de Modelagem Matemática, ofertada para graduandos do curso de Matemática de dois estados distintos, que não haviam conseguido aprovação na primeira vez. Os da segunda obra descreveram atividades online, que ocorriam como parte da carga horária de disciplinas presenciais, em que os docentes orientavam, supervisionavam e avaliavam o progresso dos graduandos. Já o trabalho de Parra-Zapata et al. (2018) teve direcionamento para a formação continuada com um curso desenvolvido com nove professores brasileiros e oito colombianos.

Diferente do que aconteceu nos trabalhos citados até então, em Schroetter et al. (2016) houve a participação de alunos da educação básica. As autoras diferenciaram-se também ao 
usarem o e-mail como AVA, para comunicação assíncrona com os alunos. O uso do e-mail se deu mediante a inexistência de uma plataforma gerida pela escola, que acolhesse o desenvolvimento da atividade. Em Orey e Rosa (2018), por outro lado, houve a opção por uma comunicação híbrida, alternando entre encontros síncronos via videoconferências, e assíncronos, na Plataforma Moodle. Esse hibridismo também foi pontuado por Parra-Zapata et al. (2018), em que houve um encontro síncrono de quatro horas na plataforma WizlQ e duas sessões assíncronas, uma antes e outra depois do encontro síncrono, viabilizadas na Plataforma Moodle e no Google Drive.

A Plataforma Moodle também foi utilizada no relato de Borssoi, Silva e Ferruzzi (2017), na qual ancorava um Ambiente Virtual de Ensino e Aprendizagem (AVEA), no entanto, dificuldades dos grupos de graduandos em interagir nesse ambiente acabaram fazendo com que eles recorressem a outros espaços de interação, como o aplicativo WhatsApp. Infelizmente em Borssoi, Silva e Ferruzzi (2017) não encontrei descrições de uma atividade de Modelagem Matemática desenvolvida pelos graduandos no AVEA do começo ao fim, o que dificultou entender como seria essa dinâmica. Porém, os recortes de diferentes atividades apresentados e discutidos da interação dos graduandos com as professoras mostram que as atividades focavam no aprendizado de conteúdos matemáticos, conforme se realizavam as fases de Modelagem Matemática (ALMEIDA; SILVA; VERTUAN, 2012).

Já nos trabalhos de Schroetter et al. (2016), Orey e Rosa (2018) e Parra-Zapata et al. (2018), considerando os objetivos de pesquisa assumidos nos trabalhos, foi possível entender a dinâmica de suas atividades de Modelagem Matemática, a partir de suas descrições.

$\mathrm{Na}$ atividade de Modelagem Matemática relatada por Schroetter et al. (2016) os alunos foram separados em grupos, e para cada foi criado um e-mail em que ocorreram as mediações entre os alunos e as pesquisadoras. A atividade requeria conteúdos matemáticos já vistos, iniciada a partir de um problema gerador (forrar uma caixa de papelão) e de três questões iguais para todos os grupos. Questão 1. Quanto papel será necessário? 2. Sabe-se que a folha de papel pardo medindo $80 \mathrm{~cm} \times 120 \mathrm{~cm}$ custa $\mathrm{R} \$ 1,00$. Quanto será gasto em reais para forrar a caixa? 3 . Dividindo-se o valor gasto para forrar a caixa entre os integrantes do seu grupo, quanto cada um terá que desembolsar? Posteriormente foi proposto aos alunos uma quarta questão, que solicitava de cada grupo que determinasse a quantidade de certo produto que poderia ser colocado dentro da caixa de papelão. Os autores entendiam a atividade de Modelagem Matemática como sendo uma metodologia de ensino e aprendizagem (BIEMBENGUT; HEIN, 2000; STAHL, 2003, MEYER; CALDEIRA; MALHEIROS, 2011). 
Orey e Rosa (2018) descrevem projetos de Modelagem Matemática (ROSA, 2000), em que foram investigados os temas: poluição da água, do ar, e consumo de água. Com ferramentas da plataforma Moodle os tutores e professores postavam encaminhamentos para a disciplina, e os alunos postavam textos, impressões, dados acerca dos temas investigados. Os alunos aproveitavam os encontros síncronos para tirar dúvidas referentes às fases dos projetos e sobre os conteúdos matemáticos.

A investigação de diferentes temáticas também foi pontuada em Parra-Zapata et al. (2018) a respeito do curso envolvendo temas como: educação à distância, tecnologias digitais, visualização, formação de professores, modelagem matemática e evolução das matemáticas. Nesse contexto Parra-Zapata et al. (2018) definem a Modelagem Matemática como uma alternativa para promover formas diferentes de participação e auxiliar no desenvolvimento de atitudes crítica e criativa (PARRA-ZAPATA et al., 2017).

Ao observar o que foi discutido até aqui nos artigos de periódicos e de anais de eventos, identificamos pontos que corroboram as afirmações de Menezes (2019), como atividades de Modelagem Matemática no contexto online, voltados para a formação inicial ou continuada. No entanto, houve também informações inéditas, como o desenvolvimento de atividades de Modelagem Matemática via e-mail, de forma assíncrona, para o ensino de alunos da educação básica. Na próxima seção, discutimos os pontos encontrados por Menezes (2019), juntamente com os itens estudados na análise dos artigos de periódicos e de anais de eventos.

\section{Direcionamentos encontrados nos trabalhos sobre modelagem matemática no contexto online}

Considerando nosso objetivo de descrever um mapeamento de pesquisas que abordaram o desenvolvimento de atividades de Modelagem Matemática de forma online, observamos nos trabalhos analisados que houve uma mudança nas pesquisas quanto ao propósito, quando analisados os trabalhos oriundos dos periódicos e dos anais de evento. Ainda foram encontrados trabalhos que abordavam o desenvolvimento de atividades de Modelagem Matemática no contexto online, como opção metodológica na formação de professores, como no caso de Parra-Zapata et al. (2018), que relata o desenvolvimento de tarefas de Modelagem Matemática nas plataformas WizIQ e Moodle. Contudo, foram encontrados trabalhos que priorizaram tanto formação em práticas de Modelagem Matemática como metodologia, quanto o ensinar Matemática por meio da Modelagem Matemática. Como no caso de Orey e Rosa (2018), que relata o desenvolvimento de 
projetos por graduandos de um curso na plataforma Moodle, em que, ao mesmo tempo em que eram informados acerca das fases e subfases da Modelagem Matemática, também aprendiam conteúdos matemáticos ao enxergá-los nos contextos reais dos temas investigados nos projetos.

Ainda imerso nessa tipificação de propósitos, destaco trabalhos que relataram o uso da Modelagem Matemática com o propósito principal de ensinar Matemática. Como no caso de Borssoi, Silva e Ferruzzi (2017), que sinalizaram o desenvolvimento de atividade no AVEA em disciplinas como Cálculo Diferencial e Integral, Cálculo Numérico entre outras, e o trabalho de Schroetter et al. (2016), que relataram o desenvolvimento de atividades de Modelagem Matemática por e-mail, com alunos da educação básica. Assim, considerando os textos analisados, identifiquei que existiu uma predominância de trabalhos em que a Modelagem Matemática no contexto online, foi apresentada/investigada como uma possibilidade para formação inicial ou continuada de professores que ensinam matemática. No entanto, verificamos nos últimos anos, trabalhos em que a Modelagem Matemática no contexto online é utilizada para ensinar Matemática, mostrando-se também uma vertente frutifera a ser pesquisada.

Como o mapeamento em Menezes (2019) tratou de dissertações e teses, e ampliamos a cobertura do mapeamento incluindo artigos de periódicos e de anais de eventos, organizamos os trabalhos mapeados por Menezes (2019) destacados em azul, e os artigos de periódicos e anais destacados em laranja, segundo dois perfis no infográfico: Formação - atividades de Modelagem Matemática no contexto online como referência metodológica, para formação inicial ou continuada de professores que ensinam Matemática, em que o foco é entender o processo de Modelagem Matemática; Ensino - atividades de Modelagem Matemática no contexto online para ensinar Matemática, independente de se os alunos sabem ou não que estão vivenciando uma atividade de Modelagem Matemática, já que o foco está na aprendizagem de conteúdos matemáticos. Como mostra a Figura 1. 
Figura 1: Infográfico com os trabalhos organizados segundo os perfis Formação (azul) e Ensino (laranja)

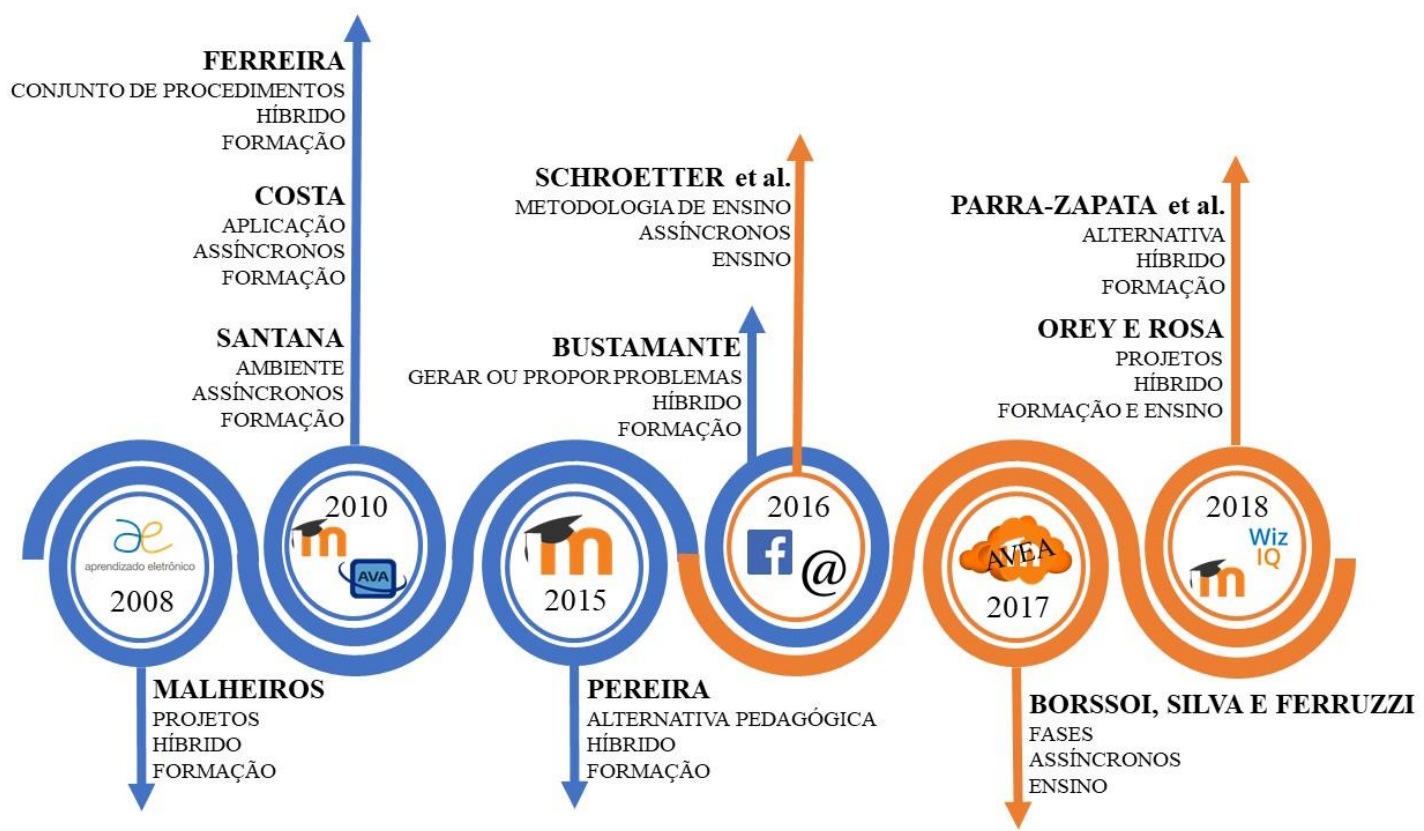

Fonte: Autores, 2021

O número de trabalhos analisados, quando comparado com o número de trabalhos de Modelagem Matemática que foram investigados neste artigo, é pequeno, sendo o contexto de ambos os perfis frutíferos para novas pesquisas. No entanto, o perfil Ensino mostra-se tímido em relação ao perfil Formação, e nesse sentido apresenta potencial para novas pesquisas.

Além disso, outra característica que permeou os trabalhos de ambos os perfis foram os tipos de encontros no desenvolvimento das atividades de Modelagem Matemática no contexto online, nos quais foi percebido que as interações entre professores e alunos nos AVA foram estabelecidas de forma assíncrona ou por um modelo híbrido, no qual se mesclou encontros síncronos e assíncronos. Não foram encontrados relatos de propostas de atividades de Modelagem Matemática desenvolvidas em sua totalidade por meio de comunicação síncrona. Ao detectar essa lacuna, esse mapeamento é importante na pesquisa de doutorado do primeiro autor cujo propósito foi investigar interações que apareceram entre estudantes, mediador e conteúdos matemáticos durante o desenvolvimento síncrono de tarefas de Modelagem Matemática, em um ambiente online de ensino e aprendizagem.

Não tivemos a intenção de fazer um trabalho de estado da arte sobre trabalhos que investigaram atividades de Modelagem Matemática de forma online. Reconhecemos algumas limitações do trabalho, no sentido de estar condicionado as escolhas do primeiro autor que se movimentava as justificando ora amparado em argumentos científicos e ora em escolhas pessoais, e que dessa forma, outros mapeamentos podem resultar em outros achados. No entanto, como 
propõe Biembengut (2008) entendemos esse mapeamento como sendo o primeiro passo da pesquisa, na qual essa revisão de literatura objetivava identificar e reconhecer o campo no qual 0 primeiro autor encontrou o objeto de investigação da sua pesquisa de tese de doutorado, mostrando-se assim, um estudo profícuo para pesquisadores iniciantes.

\section{Referências}

ALMEIDA, L. M. W. de; SILVA, K. P. da; VERTUAN, R. E. Modelagem Matemática na educação básica. São Paulo: Contexto, 2012.

BARBOSA, J. C. A dinâmica das discussões dos alunos no ambiente de Modelagem Matemática. In: $3^{\circ}$ SEMINÁRIO INTERNACIONAL DE PESQUISA EM EDUCAÇÃO MATEMÁTICA, 2006, Águas de Lindóia. Anais... Recife: SBEM, 2006. 1 CD-ROM.

BARBOSA, J. C. As relações dos professores com a Modelagem Matemática. In: $8^{\circ}$ ENCONTRO NACIONAL DE EDUCAÇÃO MATEMÁTICA, ENEM, 2004, Recife. Anais... Recife: SBEM, 2004. 1 CD-ROM.

BARBOSA, J. C. Modelagem Matemática e a perspectiva sócio-crítica. In: $2^{\circ}$ SEMINÁRIO INTERNACIONAL DE PESQUISAS EM EDUCAÇÃO MATEMÁTICA, 2003, Santos. Anais... São Paulo: SBEM, 2003b. CD-ROM.

BORSSOI, A. H.; SILVA, K. A. P.; FERRUZZI, E. C. Modelagem matemática no ambiente virtual de ensino e aprendizagem: alguns encaminhamentos. In: 10 ${ }^{a}$ Conferência Nacional sobre Modelagem na Educação Matemática, 2017, Maringá. Anais... Maringá, 2017.

BASSANEZI, R. C. Ensino-aprendizagem com Modelagem Matemática. São Paulo: Contexto, 2002.

BIEMBENGUT, M. S. Mapeamento na Pesquisa Educacional. Rio de Janeiro: Ciência Moderna Ltda., 2008.

BIEMBENGUT, M. S.; HEIN, N. Modelagem Matemática no ensino. São Paulo: Contexto, São Paulo, 2000.

BORBA, M. C.; VILLARREAL, M. E. Humans-With-Media and the Reorganization of Mathematical Thinking: information and communication technologies, modeling, experimentation and visualization. New York: Springer, 2005. v. 39.

BURAK, D. Modelagem matemática e a sala de aula. In: $1^{\circ}$ Encontro Paranaense de Modelagem em Educação Matemática, 2004, Londrina. Anais... Londrina, 2004.

BURAK, D. Modelagem matemática: uma alternativa para o ensino de matemática na 5 a série. 1987. Dissertação (Mestrado em Educação Matemática) - Universidade Estadual Paulista, Rio Claro, 1987.

BUSTAMANTE, J. E. G. Modelagem matemática na modalidade online: Análise segundo a teoria da atividade. 2016. 213f. Tese (Doutorado em Educação Matemática) - Instituto de 
Geociências e Ciências Exatas, Universidade Estadual Paulista "Júlio de Mesquita Filho" - UNESP. Rio Claro, 2016.

COSTA, P. O. Educação on-line na Universidade: o processo de ensinar e aprender cálculo na era das tecnologias digitais. 2010. 145f. Dissertação (Mestrado em Ciências Humanas) Universidade Federal de Uberlândia - UFU, Uberlândia, 2010.

FERREIRA, C. R. Modelagem matemática na Educação Matemática: contribuições e desafios à Formação Continuada de professores na modalidade Educação à Distância online. 2010. 101f. Dissertação (Mestrado em Educação) - Universidade Estadual de Ponta Grossa, Ponta Grossa, 2010.

FERREIRA, C. R.; BURAK, D. Modelagem Matemática: uma experiência com a formação continuada de professores utilizando a educação a distância online. In: $10^{\circ}$ Encontro Nacional de Educação Matemática, 2010, Salvador, Anais... Salvador, 2010.

FERREIRA, C. R.; BURAK, D. Considerações Iniciais sobre um Curso de Modelagem Matemática em Ambientes Virtuais. In: $6^{a}$ CONFERÊNCIA NACIONAL SOBRE MODELAGEM NA EDUCAÇÃO MATEMÁTICA, 2009, Londrina. Anais... Londrina, 2009.

FREITAS, J. de F. R. B.; OREY, D. C.; ROSA, M. Propondo a Modelagem Matemática como um Ambiente Virtual de Aprendizagem. In: $10^{\text {a }}$ CONFERÊNCIA NACIONAL SOBRE MODELAGEM NA EDUCAÇÃO MATEMÁTICA, 2017, Maringá. Anais... Maringá, 2017.

GALLEGUILLOS, J. Proposta e resolução de um problema de modelagem na Educação Matemática online. In: $12^{\circ}$ Encontro Nacional de Educação Matemática, 2016, São Paulo. Anais... São Paulo, 2016.

GALLEGUILLOS, J.; BORBA, M. D. C. Expansive movements in the development of mathematical modelling: analysis from an activity theory perspective. ZDM Mathematics Education, v. 50, n. 1-2, p. 129-142, 2018.

MALHEIROS, A. P. dos S. Projetos de Modelagem em um curso de formação on-line. In: $4^{0}$ SEMINÁRIO INTERNACIONAL DE PESQUISA EM EDUCAÇÃO MATEMÁTICA, 2009, Taguatinga. Anais... Taguatinga, 2009.

MALHEIROS, A. P. dos S. Educação Matemática online: a elaboração de projetos de Modelagem. 2008. 186 f. Tese (Doutorado em Educação Matemática) - Instituto de Geociências e Ciências Exatas, Universidade Estadual Paulista "Júlio de Mesquita Filho" - UNESP. Rio Claro, $2008 \mathrm{a}$

MALHEIROS, A. P. dos S. Educação Matemática online: a elaboração de projetos de Modelagem. Bolema - Revista Boletim de Educação Matemática, Rio Claro, v. 21, n. 30, p. 208-209, 2008b.

MATUCHESKI, S. Educação Matemática online: a elaboração de projetos de Modelagem. Bolema - Revista Boletim de Educação Matemática, Rio Claro, v. 26, n. 42B, p. 763-766, 2012.

MENEZES, R. O. Mapeamento em Dissertações e Teses de Atividades de Modelagem Matemática Desenvolvidas na Modalidade a Distância. In: 39ª REUNIÃO NACIONAL ANPEd, 2019. Anais... Niterói-RJ, 2019. 
MEYER, J. F. C. A.; CALDEIRA, A. D.; MALHEIROS, A. P. S. Modelagem em Educação Matemática. Coleção Tendências em Educação Matemática. Belo Horizonte: Autêntica, 2011.

OREY, D. C.; ROSA, M. Developing a mathematical modelling course in a virtual learning environment. ZDM - The International Journal on Mathematics Education, v. 50, n. 1-2, p. 173$185,2018$.

PARRA-ZAPATA, M. M. et al. Participación de profesores en un ambiente de formación online. Un estudio en modelación matemática. Educación Matemática, México, Distrito Federal, v. 30, n. 1 , p. 185-212, abr. 2018.

PARRA-ZAPATA, M. M., Rendón-Mesa, P. A., Molina-Toro, J. F., Sánchez-Cardona, J., Ocam ᄀpoArenas, M. C., Villa-Ochoa, J. A. Participación de profesores en un ambiennte de formación online. Ejemplo de un diseño en modelación matemática. Revista Virtual Universidad Católica del Norte, 50, p. 3-20, 2017.

PEREIRA, R. S. G. A educação a distância e a formação continuada de professores de matemática: contribuições de um contexto formativo para a base de conhecimento docente (Tese de Doutorado) - Universidade Estadual Paulista Júlio de Mesquita Filho/Presidente Prudente, 2015.

RANDOLPH, J. J. A Guide to Writing the Dissertation Literature Review. Practical Assessment Research Evaluation, v.14, 2009.

ROSA, M. From reality to mathematical modelling: A proposal for using ethnomathematical knowledge. College of Education. Sacramento: California State University, Sacramento (CSUS), 2000.

SANTANA, T. Analisando aspectos da organização de um curso de Modelagem a distância: uma contribuição para o debate da pedagogia on-line na educação matemática. In: $8^{a}$ CONFERÊNCIA NACIONAL SOBRE MODELAGEM NA EDUCAÇÃO MATEMÁTICA, 2013, Santa Maria. Anais... Santa Maria, 2013.

SANTANA, T. Avaliação discente de um curso de modelagem matemática à distância. 2010. 122f. Dissertação (Mestrado em Ensino, Filosofia e História das Ciências) - Universidade Federal da Bahia, Universidade Estadual de Feira de Santana, Salvador, 2010.

SCHROETTER, S. M.; STAHL, N. S.; CHRYSOSTOMO, C. S.; DUNCAN, C. R. A escrita e o pensamento matemático no ambiente virtual utilizando a modelagem matemática: experiência de uma turma de $9^{\circ}$ ano. Educação Matemática Pesquisa, São Paulo, v.18, n.1, p. 373-396, 2016.

STAHL, N. S. P. O Ambiente e a Modelagem Matemática no Ensino do Cálculo Numérico. Campinas. Tese (Doutorado em Educação Matemática) - Faculdade de Educação, Unicamp, Campinas, 2003. 